\title{
50 AÑOS DE CIRUGÍA DE BYPASS CORONARIO. Meditar el pasado, enfrentar el presente y forjar el futuro
}

\author{
Ricardo Zalaquett \\ División de Enfermedades Cardiovasculares \\ Facultad de Medicina Pontificia Universidad Católica de Chile
}

Resumen: Al cumplirse este año 50 años de la cirugía de bypass coronario, introducida y desarrollada por René Favaloro en la Cleveland Clinic, es conveniente meditar el camino seguido previamente por la cirugía para tratar la enfermedad coronaria, analizar cómo la cirugía enfrenta en la actualidad esta grave y frecuente enfermedad, y, si es posible, predecir el rol que ésta jugará en el futuro en el tratamiento de la enfermedad coronaria. 


\section{Fifty years of Coronary Artery Surgery}

This year is the 50th anniversary of Coronary Artery Bypass Graft surgery, introduced and developed by René Favaloro at The Cleveland Clinic. The occasion calls for meditation about the path followed by surgery to treat coronary artery disease in the past, to analyze how surgery currently faces this serious and frequent disease and, if possible, to predict the role that surgery will play in the future in the treatment of coronary artery disease.

Keywords: Coronary artery disease, cardiac surgery, aorto-coronary bypass, history. 
El 9 de mayo de 1967, René Favaloro efectuó, en la Cleveland Clinic, una interposición de un segmento invertido de vena safena en la arteria coronaria derecha ocluida de una paciente de 57 años con angina crónica. Este no fue un procedimiento fortuito o producto de la urgencia del momento, sino que completamente planeado, luego de analizar, junto a Mason Sones, la coronariografía de la paciente (de hecho, la paciente fue reestudiada por este último al octavo día, demostrándose una reconstrucción total de la arteria). Tampoco fue un procedimiento aislado, sino que fue seguido por otros 13 casos programados, hasta que en el paciente número 15 Favaloro efectuó, el 19 de octubre de ese mismo año, un puente aorto-coronario con vena safena propiamente tal. En abril de 1968, Favaloro publicó sus resultados en los 15 primeros pacientes y mencionó otros 40 en un apéndice. Había nacido así la CIRUGÍA DE BYPASS CORONARIO, la que fue rápidamente respaldada por W. Dudley Johnson, en Milwaukee y George Green, en Nueva York, confirmando el valor de esta para el tratamiento de la enfermedad coronaria. La operación fue rápida y entusiastamente adoptada por los cirujanos de todo el mundo, si bien inicialmente no tanto por los cardiólogos, efectuándose solo en Estados Unidos más de 100.000 casos en 1977 y más de 600.000 en 1997.

\section{El pasado}

Favaloro llegó directamente a la Cleveland Clinic, en sus propios términos, "de la pampa a los Estados Unidos" en enero de 1962, con 39 años cumplidos, con la idea de convertirse en un cirujano cardiotorácico, portando sólo una carta de recomendación de su antiguo profesor de cirugía (Figura 1). A pesar de sus problemas iniciales con el inglés, fue rápidamente acogido por Donald Effler, Jefe de Cirugía Torácica y por Bernie Crile, jefe del Departamento de Cirugía, logrando ser aceptado como residente primero y luego como staff de la Cleveland Clinic. Durante este periodo Favaloro ganó experiencia con los procedimientos coronarios de la época, como el Implante Mamario (operación de Vineberg), la endarterectomía y el parche coronarios. En 1966, inspirado por el análisis de múltiples coronariografías junto a Mason Sones (Figura 2) y por su relación con los cirujanos vasculares de la Cleveland Clinic, en especial con el chileno Edwin Beven, con los que aprendió de los injertos de vena safena para la revascularización de los riñones y las extremidades inferiores, su interés se volcó hacia el desarrollo del bypass aorto-coronario (Figura 3).

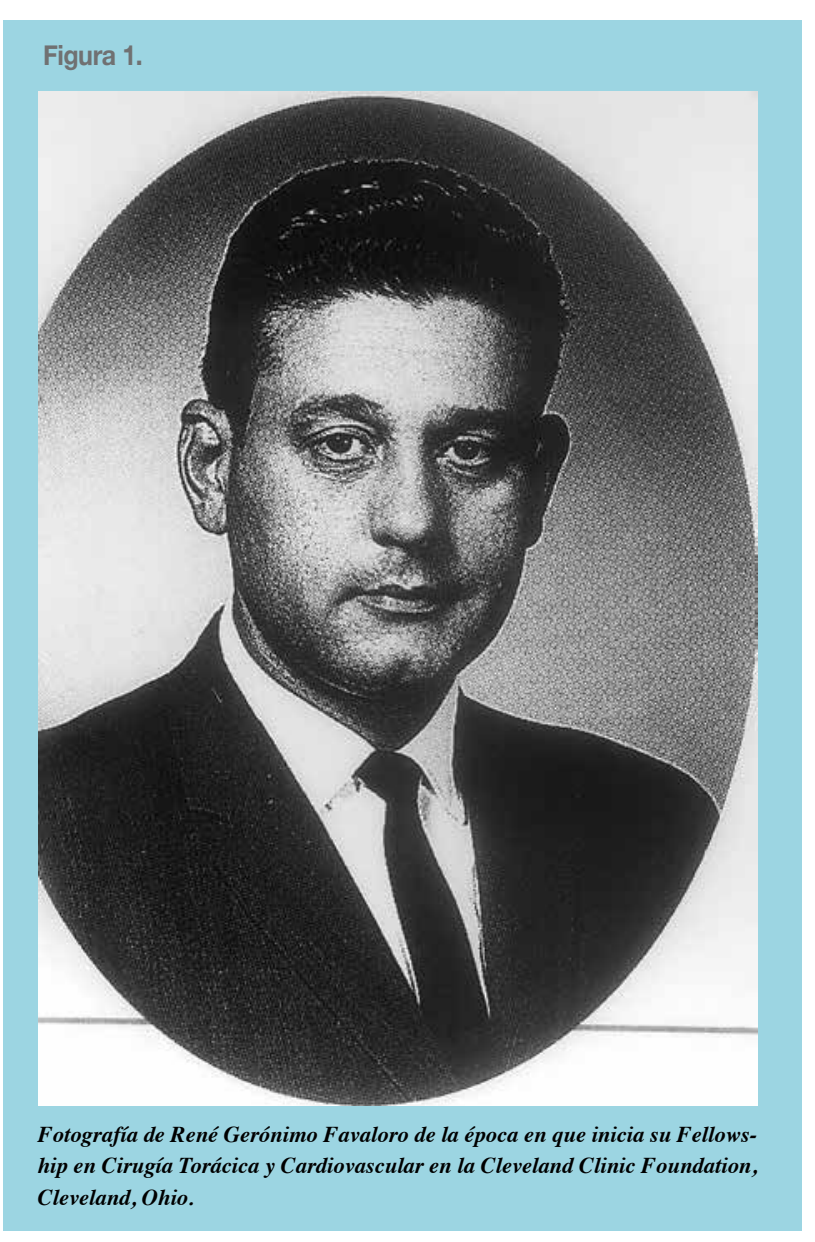

Figura 2

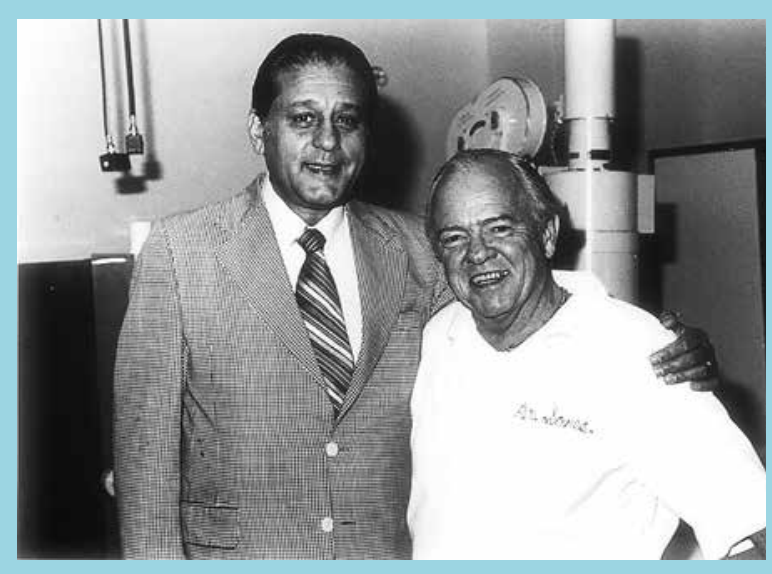

René Favaloro (izquierda) y Mason Sones (derecha). Foto tomada en el Laboratorio de Hemodinamia de la Cleveland Clinic.

Es interesante conocer que las bases experimentales del bypass aorto-coronario, si bien escasas, se remontan a 1910. Ese año, Alexis Carrel, de hecho, el fundador de la cirugía vascular y premio Nobel 1912 por sus tra- 


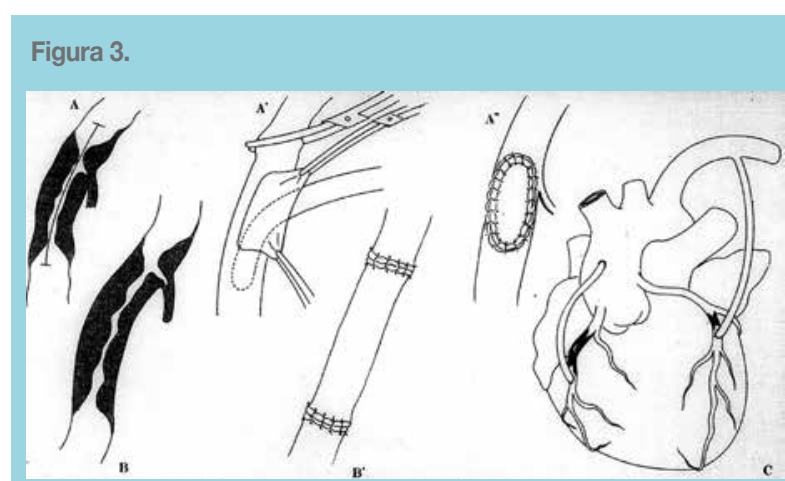

Representación esquemática de la evolución de la cirugía coronaria, según Favaloro en su libro autobiográfico "De la Pampa a los Estados Unidos".

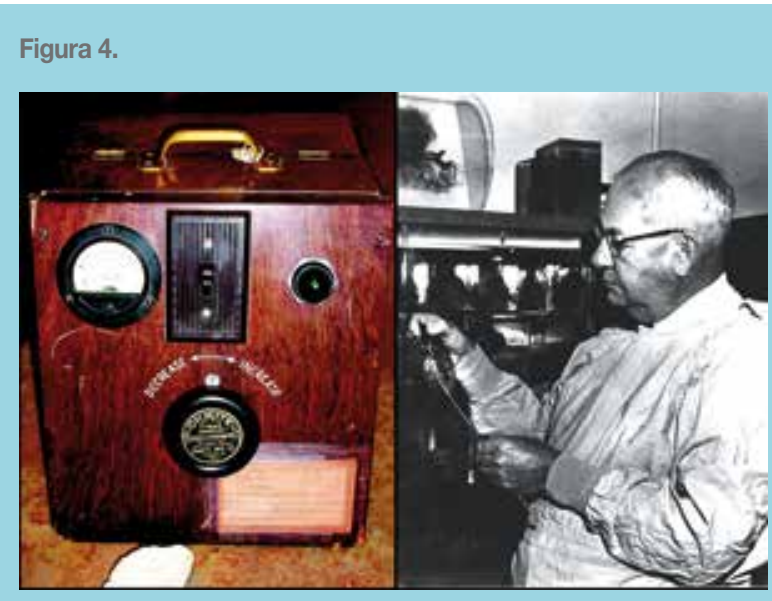

Claude S. Beck (1894-1974). Beck no sólo fue un gran pionero de los procedimientos indirectos para aumentar la circulación coronaria, sino que también en el desarrollo de la desfibrilación miocárdica y del masaje cardíaco para la resucitación cardiopulmonar.

bajos al respecto, efectuó en un perro un bypass entre la aorta descendente y las arterias coronarias utilizando un segmento de arteria carótida preservado en frío. Sin embargo, el experimento fracasó, puesto que el perro desarrolló una fibrilación ventricular, de la cual murió. Como quiera que sea, es necesario reconocer que Carrel ya ese año concibió la idea del bypass aorto-coronario, por lo que para algunos la cirugía coronaria debiera estar celebrando 107 años. Además, de su experimento concluyó que el procedimiento habría sido exitoso si hubiera sido capaz de efectuar la anastomosis en menos de 3 minutos, y no en los 5 que se demoró (a este respecto, es necesario recordar que la "rapidez" ha estado siempre presente en la cirugía coronaria, primero, cuando ésta se hacía, en circulación extracorpórea, pero con clampeo aórtico intermitente, el que no debía superar los 10 a 15 minutos, y, hace algunos años cuándo se puso muy en boga la cirugía coronaria sin bomba).
En los años 40 y 50, Gordon Murray, en Toronto, experimentó en animales, a los cuales resecaba segmentos de las coronarias, interponiendo luego injertos o efectuando puentes coronarios con diferentes arterias. Desafortunadamente, los animales desarrollaban fibrilación ventricular o trombosis de los injertos con gran frecuencia.

Descorazonados con los resultados de Carrel y Murray, los cirujanos siguieron otros caminos para tratar la enfermedad coronaria en los años siguientes.

Procedimientos extracardíacos o indirectos. En 1930 Blumgart y colaboradores, a partir de la observación clínica de la tirotoxicosis, dedujeron que disminuyendo las demandas metabólicas del organismo debía disminuir el trabajo cardíaco. De aquí que la tiroidectomía total debería mejorar la insuficiencia cardíaca y la angina de pecho, lo que efectuaron en 10 pacientes. Posteriormente, esta fue sustituida por yodo radiactivo. Ambos procedimientos fueron rápidamente abandonados por la gran depresión metabólica que producían.

Otro procedimiento fue la simpatectomía bilateral, con la cual se perseguía no solo aliviar la angina, sino que también producir vasodilatación coronaria, efectuada a través de distintas técnicas, entre ellas, la inyección paravertebral de alcohol. Con un objetivo similar, en 1930 se introdujo la irradiación del corazón.

Procedimientos indirectos para aumentar la irrigación del corazón. Posteriormente, entre los años 1935 y 1953, se diseñaron procedimientos quirúrgicos sobre el corazón con el objeto de inducir una neovascularización y aumentar la circulación colateral al miocardio. Así, el corazón fue erosionado, irritado, inflamado, envuelto, denervado, perforado, el drenaje venoso ligado y arterializado, etcétera. Con este fin, el corazón fue cubierto, parcial o totalmente, con epiplón (cardioomentopexia), pulmón (cardioneumopexia), yeyuno (cardioyeyunopexia), estómago (cardiogastropexia), bazo (cardioesplenopexia) músculo (pectoral mayor o dorsal ancho), tejido retro esternal, grasa pericárdica, pedículo cutáneo, etcétera.

En este periodo destaca la imponente figura de Claude Beck, cirujano de Case Western Reserve University y The Cleveland Clinic, en Cleveland, Ohio (Figura 4). Originalmente, un neurocirujano, en 1930 Beck comenzó a estudiar los efectos de la compresión del corazón por cicatrices y adherencias inducidas experimentalmente en animales. En 1932 se enteró de las publica- 
ciones de Moritz sobre colaterales entre los territorios vasculares y de la vascularización de las adherencias pericárdicas. En 1934, Beck se percató del sangramiento activo al seccionar cicatrices pericárdicas en ambos extremos. A partir de estas observaciones, Beck desarrolló diferentes procedimientos para incrementar las anastomosis vasculares entre las arterias coronarias y tejidos adyacentes. El primero de estos procedimientos consistió en producir una pericarditis estéril por abrasión mecánica de ambas hojas pericárdicas, con el objeto de producir adherencias vasculares que se comunicaran con los vasos miocárdicos. Este procedimiento, que se conoció como pericardiopexia o poudrage pericárdico, sería el primero de una larga serie desarrollados por Beck durante su carrera. Los próximos 20 años, Beck experimentó con diferentes irritantes para producir adherencias aún más efectivas, como hueso triturado, arena, talco, fenol, nitrato de plata, Ivalón e, incluso, asbesto, entre otros.

Entre los procedimientos más sorprendentes desarrollados en este periodo está la "ligadura bilateral de las arterias mamarias". El objetivo supuesto de este procedimiento era incrementar el flujo sanguíneo al corazón a través del desarrollo de comunicaciones extra cardíacas entre las arterias coronarias y las arterias epicardiofrénicas. Dada su simplicidad, este procedimiento alcanzó una rápida pero corta popularidad, siendo precozmente desacreditada al demostrase que operaciones "sham", sin ligadura de las arterias mamarias, igualmente aliviaban la angina, eso sí, por un corto periodo de tiempo. Sin embargo, en años sucesivos, esta operación fue revivida en varias oportunidades, especialmente por Glover, en 1958, quien había sido ayudante de Bailey en las primeras comisurotomías mitrales, quien sostenía que la operación "era tan simple y libre de riesgos, que valía la pena intentarla".

Sin embargo, el procedimiento quirúrgico más importante y de más larga vida desarrollado en este periodo fue el Implante de Arteria mamaria Interna, conocido como "Operación de Vineberg". Este fue un procedimiento completamente original para crear un aporte nuevo y diferente de sangre al corazón, desarrollado experimentalmente en 1946 por Arthur M. Vineberg, en McGill University, en Montreal (Figura 5). Este consistía en el autotrasplante de la arteria mamaria interna izquierda, a través de una toracotomía anterolateral, introduciendo el extremo abierto de esta arteria en un túnel en la pared anterior del ventrículo izquierdo, con el fin de desarrollar colaterales que se conectaran con

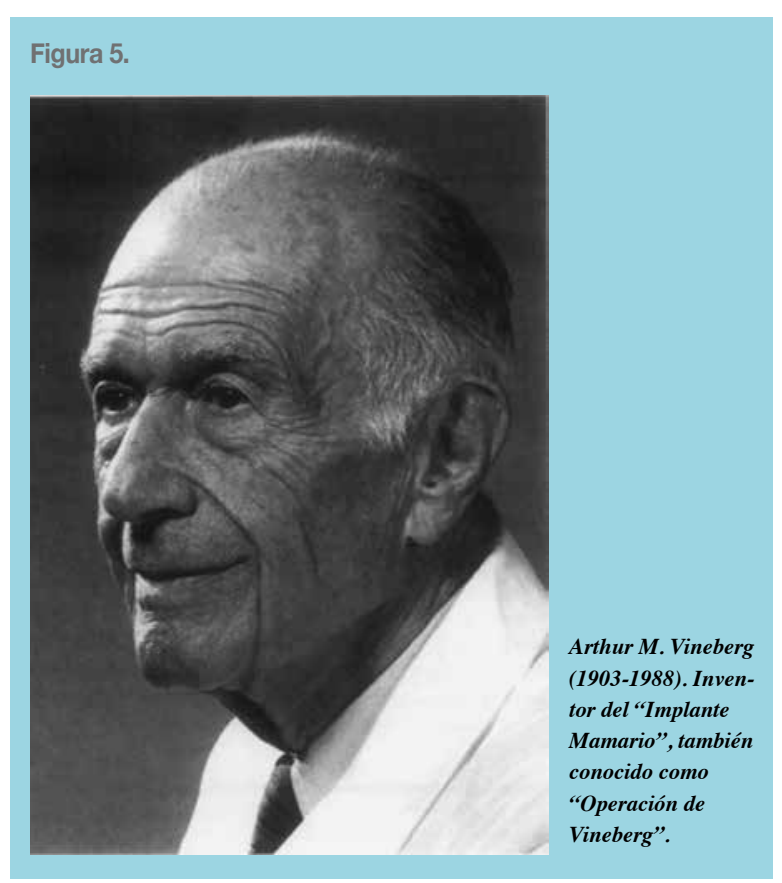

el árbol coronario. Vineberg efectuó el primer procedimiento en humanos en 1950, comunicando en 1958 sus resultados en 59 pacientes, con buenos resultados clínicos en cuanto al alivio de la angina, definiendo, además, que la operación estaba indicada sólo en pacientes con angina estable, puesto que en caso contrario la mortalidad operatoria era muy elevada. A pesar del entusiasmo de Vineberg, la comunidad cardiovascular se mantuvo escéptica, hasta 1962 en que Sones estudió angiográficamente 2 pacientes operados por Vineberg, demostrando, en vivo, comunicaciones vasculares entre el implante de arteria mamaria y el sistema coronario. Effler, Jefe de Cirugía Cardiovascular de la Cleveland Clinic, se entusiasmó con la operación comunicando, en 1963, 46 casos favorablemente operados con la técnica de Vineberg. El implante mamario se continuó efectuando hasta fines de los años 70, e incluso comienzos de los 80, siendo Favaloro uno de sus grandes proponentes (Favaloro en un comienzo no fue entusiasta del injerto de arteria mamaria interna a la arteria descendente anterior, entre otras, porque impediría el implante mamario).

Procedimientos iniciales directos sobre la circulación coronaria. Hasta 1956, como los que hemos señalado, los procedimientos quirúrgicos para tratar la enfermedad coronaria estuvieron dirigidos a aumentar indirectamente el flujo sanguíneo al miocardio o disminuir sus requerimientos metabólicos. De hecho, Beck, a partir de 
sus trabajos experimentales, pensaba que cualquier procedimiento sobre la circulación coronaria propiamente tal podría originar un disturbio eléctrico del corazón, que haría más susceptible a los ventrículos a fibrilar. Sin embargo, ese mismo año 1956, Lillehei y colaboradores, en la University of Minnessota, propusieron el primer procedimiento directo para el tratamiento de la enfermedad coronaria: La endarterectomía coronaria.

Endarterectomía coronaria. Lillehei y colaboradores observaron que en la mayoría de los pacientes las oclusiones de las arterias coronarias se producían en los 4 centímetros proximales de éstas, por lo que estas placas ateroscleróticas obstructivas podrían ser removidas, o, en caso contrario, se podría efectuar una resección segmentaria de la arteria.

La primera endarterectomía coronaria en un paciente fue efectuada en 1956 por Bailey (quien había efectuado ya la primera comisurotomía mitral en 1948) en Filadelfia. Posteriormente, Longmiere, en California, hizo varias comunicaciones de importancia, antes de dedicarse por completo a la cirugía digestiva. En 1961, Senning complementó la endarterectomía coronaria con un parche de vena safena, lo que fue modificado por Effler y colaboradores, entre ellos Favaloro, utilizando un parche de pericardio autólogo, procedimiento que ganó gran aceptación (la verdad es que el uso de pericardio fue fortuito, ya que la vena safena que se iba a utilizar como parche se cayó accidentalmente al suelo). La operación en este caso se efectuaba con circulación extracorpórea e hipotermia sistémica, a diferencia de los casos iniciales de endarterectomías.

\section{El presente}

A diferencia de los procedimientos efectuados en el pasado, el puente o bypass aorto-coronario no fue precedido por una investigación experimental en animales por parte de Favaloro, si bien éste estaba al tanto de los trabajos de este tipo efectuados por Gordon Murray en Toronto en los años 40 y 50. Sin embargo, tampoco fue el resultado de un hecho fortuito, como se ha señalado en la introducción. El bypass aorto-coronario fue la consecuencia de la extensa experiencia previa de Favaloro con la endarterectomía coronaria, con el parche coronario y los implantes mamarios, así como como de la interrelación de éste con los cirujanos vasculares de la Cleveland Clinic pero, por sobre todo, de la estrecha amistad con Mason Sones, lo que le permitió el estudio y análisis de múltiples coronariografías, situaciones to- das facilitadas por el ambiente académico y clínico de la Cleveland Clinic.

Favaloro no fue el primer cirujano en efectuar un puente aorto-coronario, ya que previamente hubo casos aislados y anecdóticos, entre los cuales los más conocidos son los de Sabiston en 1962 y Garret en 1964. Ambos casos fueron efectuados por necesidad y sin planificación previa y fueron comunicados varios años después de que Favaloro y la Cleveland Clinic hicieran tremendamente popular, académica y clínicamente, al bypass coronario.

Como en otras oportunidades, Favaloro no fue el primero en ver caer una manzana de un árbol, pero sí el primero en darse cuenta de su importancia, aplicando el puente aorto-coronario en forma sistemática, primero para la enfermedad coronaria de un vaso y, progresivamente, para la enfermedad de 2 y 3 vasos, en forma combinada con procedimientos valvulares y aneurismectomías ventriculares y en el infarto miocárdico en evolución.

En lo que respecta a la arteria mamaria interna izquierda, esta había sido utilizada experimentalmente por Murray a comienzos de los años 50 y más tarde por Spenser en los 60. Kolessov, en Rusia, había efectuado, ya en 1964, anastomosis mamario-coronarias en el hombre, no solo sin circulación extracorpórea, sino que también sin coronariografía previa. Sin embargo, fue George Green, en Nueva York, quien introdujo a partir de 1968 el uso sistemático de la arteria mamaria para la revascularización miocárdica. Green utilizaba un microscopio de 16 aumentos, lo que no sólo hacía engorrosa la operación, sino que requería de un prolongado y tedioso entrenamiento en animales, lo que dificultaba su popularización. En 1970, Favaloro, después de visitar a Green, inició el uso de la arteria mamaria interna izquierda anastomosada a la arteria descendente anterior en la forma habitual para las anastomosis coronarias en esos años, con seda 7-0 y bajo magnificación óptica corriente con lupas de 2 o 3 aumentos.

En 1986, Loop y sus colegas de la Cleveland Clinic publicaron en el New England Journal of Medicine, un trabajo, actualmente un "clásico", demostrando la superioridad del injerto de arteria mamaria interna izquierda a la arteria descendente anterior por sobre la vena safena. Esto llevó a una verdadera "fiebre" por utilizar conductos arteriales, como anastomosis únicas o secuenciales, en forma mayoritaria o exclusiva, lo que incluyó a la arteria mamaria interna derecha, la arteria gastro-epiploica, la arteria epigástrica y al "renacer" de 
la arteria radial, que había sido utilizada ya a comienzo de los años 70 por Carpentier, entre otras.

El bypass coronario fue también un precedente crucial para la angioplastia coronaria introducida por Andreas Grüntzig en 1977, la que se convirtió en el más fiero, y poco leal, competidor de aquel. En parte estimuladas por esta competencia se desarrollaron técnicas quirúrgicas "mínimamente invasivas", cuya expresión máxima es la "cirugía robótica", y se resucitó la cirugía coronaria sin circulación extracorpórea. La primera no pudo nunca competir con una punción femoral, y menos radial, y la segunda no ha sido capaz aún de demostrar una menor morbimortalidad, ni menos una mejor permeabilidad. Igualmente, se desarrollaron métodos híbridos que reunirían "lo mejor de ambos mundos": la insuperable permeabilidad de la arteria mamaria y la menor morbilidad de la angioplastia, lo que sin embargo no ha logrado aún imponerse.

Ningún procedimiento quirúrgico ha sido tan estudiado y escrutado como el bypass coronario. De hecho, esta fue la primera cirugía mayor sometida a un estudio randomizado y controlado, el primero de los cuales fue el Veterans Administration Cooperative Study publicado en el New England Journal of Medicine en 1977, seguido por el estudio Coronary Artery Surgery Study (CASS) publicado en Circulation en 1983, y luego por muchísimos más, todos los cuales demostraron el beneficio, ahora indiscutible, de la cirugía coronaria. Igualmente, con el advenimiento de la angioplastia coronaria, se iniciaron una multitud de estudios, los que continúan hasta ahora, comparando ambos procedimientos, el primero importante de los cuales fue el Bypass Angioplasty Revascularization Investigation (BARI) patrocinado por el NIH e iniciado en 1987 y que alcanzaron su máxima complejidad con el estudio SINTAX publicado en el New England Journal of Medicine en 2009, los que sólo han demostrado que la única ventaja real de la angioplastia es que no requiere una esternotomía. En 1989 la Society of Thoracic Surgeons creó un registro nacional de cirugía cardíaca, con énfasis en el bypass coronario, y el mismo año el estado de Nueva York inició la comunicación pública obligatoria de los resultados de la cirugía cardíaca. Este acucioso y demandante análisis de la cirugía coronaria continúa hasta hoy, siendo una de las mejores manifestaciones de los beneficios de la "Medicina Basada en Evidencia", en la que colaboran, como equipo, cirujanos cardíacos y cardiólogos por el bien de los pacientes.
En Chile, según Reccius en su "libro Historia y Desarrollo de la Cirugía Torácica en Chile", la primera operación para el tratamiento de la angina se efectuó en 1950 en el Hospital Van Buren de Valparaíso, por los cirujanos Svante Tornwall y Pedro Uribe, quienes realizaron una "Cardiopneumopexia" y quienes, según señalaron, en "el enfermo pudo comprobarse una recuperación extraordinaria del cuadro de insuficiencia coronaria, desapareciendo de inmediato los dolores que invalidaban al paciente, en forma absoluta."

La cirugía de bypass coronario propiamente tal se inició en Chile en 1970, en el Hospital Clínico de la Pontificia Universidad Católica de Chile. Desde entonces y hasta la fecha en nuestra institución universitaria se han efectuado más de 10.100 operaciones coronarias y se ha entrenado un grupo mayoritario de cirujanos cardiovasculares, que se han extendido a lo largo del país, contribuyendo a consolidar definitivamente la Cirugía de Bypass Coronario en Chile.

\section{El futuro}

Nada más difícil que predecir el futuro, puesto que por definición este no existe. Sin embargo, es claro que la cirugía coronaria ha ido disminuyendo en todo el mundo, en parte por los procedimientos percutáneos, que, como se ha señalado, tienen como única pero gran ventaja, que no requieren de una esternotomía, pero, principalmente, por el tratamiento médico preventivo de la enfermedad aterosclerótica. Puede ser probable que en el futuro los procedimientos quirúrgicos con robot, que no requerirían abrir la cavidad torácica, se desarrollen lo suficiente y sean rutinariamente utilizados, junto a lo cual se perfeccionen instrumentos que permitan efectuar anastomosis coronarias mecánicas y conductos artificiales que faciliten y acorten la cirugía, o que finalmente los procedimientos "híbridos" se asienten como de elección. Sin embargo, es claro que la cirugía, como siempre lo ha sido, continuará siendo la "cabeza de playa" del tratamiento de la enfermedad coronaria, en la que los pacientes van a ser cada vez de mayor edad, con más morbilidad asociada y con múltiples procedimientos previos. Y la cirugía cardíaca está ya preparada para este desafío, puesto que, si en esta ha habido un gran avance, este ha sido en la circulación extracorpórea y la protección miocárdica, las que son cada vez más eficientes y seguras, permitiendo efectuar procedimientos quirúrgicos complejos y precisos, que requieren de tiempos operatorios prolongados. 


\section{Referencias}

1- ZALAQUETT R. Del camino recorrido por la cirugía para tratar la enfermedad coronaria. Trabajo dedicado como homenaje póstumo al Dr. René Favaloro. Rev Méd Chile 2001; 129:201-208.

2- MORÁN S. Obituario. Dr. René Favaloro (1923-2000). Rev Méd Chile 2000; 128:1065-1066.

3- FAVALORO RG. De la pampa a los Estados Unidos. Buenos Aires, Argentina: Ed. Sudamericana, Octava Edición.

4- FAVALORO RG. Surgical treatment of coronary arteriosclerosis. The Williams \& Wilkins Company. Baltimore, 1970.
5- JONES DS. CABG al 50 (or 107?) - The complex course of therapeutic innovation. N Engl J Med 2017; 376:1809-1811.

6- MUELLER RL, ROSENGART TK, ISOM OW. The history of surgery for ischemic heart disease. Ann Thorac Surg 1997; 63:869-878.

7- LOOP FD, LYTLE BW, COSGROVE DM, STEWART RW, GOORMASTIC M, WILLIAMS GW et al. Influence of the internal-mammary-artery graft on 10-year survival and other cardiac events. N Engl J Med 1986; 314:1-6.

8- RECCIUS A. Historia y desarrollo de la Cirugía Torácica en Chile. Valparaíso: Imprenta y Litografía Universo, S.A., 1957. 